\title{
Optimization of Thermal Systems to Reduce Energy Consumption and Environmental Effect
}

\author{
Yogesh Jaluria \\ Board of Governors Professor and Distinguished Professor, Mechanical and Aerospace Engineering Department, Rutgers \\ University, Piscataway, New Jersey, USA
}

Corresponding Author Email: jaluria@jove.rutgers.edu

https://doi.org/10.18280/ti-ijes.630101

Received: 20 January 2019

Accepted: 18 March 2019

\section{Keywords:}

energy, environment, modelling, optimization, simulation, thermal systems

\begin{abstract}
Thermal systems, which are based on heat and mass transfer, fluid flow and thermodynamics, arise in a wide variety of applications. It has become critical to optimize thermal processes in order to reduce energy consumption and the environmental effect, while increasing the productivity and product quality. This paper discusses the optimization of thermal systems in order to achieve the best output with respect to energy and the environment. Systems from several important application areas, such as manufacturing, thermal management of electronics, and heat rejection are considered. These systems are generally quite complex due to variable material properties, uncertainties, combined transport mechanisms, complex domains and boundary conditions, and multiscale phenomena. Therefore, the modelling and simulation of these systems is quite involved and considerable care is needed to obtain accurate results. Simulation results, along with experimental data, are used for prediction of the behaviour of systems, as well as their design and optimization. The paper focuses on the reduction in energy and material consumption and the environmental effect. However, it is also important to enhance the output and improve the quality of the product obtained. The important aspects that must be considered and the approaches that may be adopted are discussed in detail. In most practical situations, several objectives are of interest and multi-objective design optimization is necessary. Results for a few important systems are presented in order to illustrate the basic approach.
\end{abstract}

\section{INTRODUCTION}

A wide range of engineering systems are based on thermal transport phenomena, which involve fluid flow, heat and mass transfer and thermodynamics. These are often known as thermal systems and are encountered in applications such as manufacturing, transportation, energy, heating/cooling, and thermal management of electronic systems, as shown schematically in Figure 1. In most practical cases, the complexity of the thermal system makes it necessary to use numerical methods to model and simulate them in order to gain information on the system behaviour and the underlying basic mechanisms. Experimentation, which is often timeconsuming and expensive, is usually employed for better physical insight and validation. The simulation results are then used to predict, control and design the system. Optimization is also necessary in most cases to enhance the efficiency of the process in order to reduce material and energy consumption and improve the product quality. It is also valuable in minimizing the environmental impact [1-2].

This paper considers the basic aspects involved in optimization of thermal systems. It discusses the modeling and numerical simulation of the relevant transport processes with realistic boundary and initial conditions that arise in practical problems. The results obtained are compared with available analytical, numerical or experimental data to verify and validate the model to achieve an accurate and physically realistic simulation. Optimization is discussed for appropriate objective functions, as well as for multi-objective optimization that applies in many cases. Of particular interest are the energy consumption, efficiency of the process, environmental effect, the quality of the product and the rate of production. Though different objective functions may be combined to yield a single objective function in a few cases, the different criteria generally need to be considered separately. This can be used to generate a Pareto frontier for nondominant solutions from which one objective function can be improved at the expense of the other. Trade-offs, based on the requirements of the problem, can then be employed to achieve the desired optimum [3]. Several practical examples are presented to illustrate the approaches that may be adopted to reduce energy consumption and enhance efficiency and product quality. The means to reduce the environmental effect of large thermal systems, such as data centers and power plant heat rejection systems, are also discussed.

\section{MODELLING AND SIMULATION}

The equations that describe thermal processes and systems are based on the conservation laws for mass, momentum and energy. A radiation source term arises for non-opaque materials like glass, which emits and absorbs energy as function of the wavelength. Also, viscous dissipation effects are important for flow of highly viscous materials like polymers and glass due to the large viscosity of the material. 
The basic equations may be written as [4]:

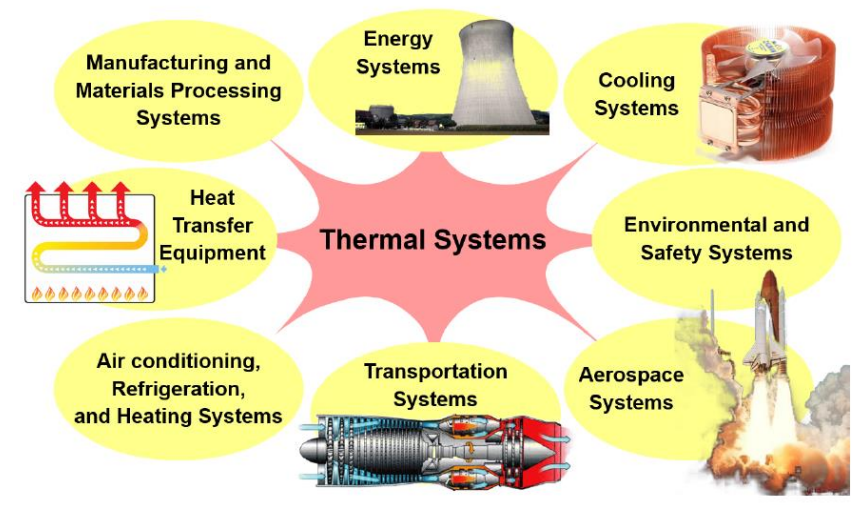

Figure 1. Examples of applications where thermal systems are of particular interest

$\frac{\mathrm{D} \rho}{\mathrm{Dt}}+\rho \nabla \cdot \overline{\mathrm{V}}=0$

$\rho \frac{\mathrm{DV}}{\mathrm{Dt}}=\overline{\mathrm{F}}+\nabla \cdot \tau$

$\rho \mathrm{C}_{\mathrm{p}} \frac{\mathrm{DT}}{\mathrm{Dt}}=\nabla \cdot(\mathrm{k} \nabla \mathrm{T})+\dot{\mathrm{Q}}+\beta \mathrm{T} \frac{\mathrm{Dp}}{\mathrm{Dt}}+\mu \Phi$

Here, $\rho, C_{p}, k$, and $\beta$ are the density, specific heat at constant pressure, thermal conductivity and coefficient of volumetric expansion, respectively, $\dot{Q}$ the thermal energy source per unit volume, $T$ the temperature, $t$ the time, $p$ the pressure, and $\bar{F}$ the body force per unit volume. $D / D t$ is the substantial or particle derivative, given in terms of the local derivatives in the flow. The stress tensor $\tau$ can be written in terms of the velocity if the fluid characteristics are known, yielding Navier-Stokes equations for common Newtonian fluids like air and water, which are often employed in cooling of electronic systems. The viscous dissipation term $\mu \Phi$ represents the irreversible part of the energy transfer due to the shear stress and is important in many practical processes such as polymer extrusion.

The relevant equations may thus be obtained for different thermal processes and systems. For instance, the equations that describe the steady flow and convective transport in many practical thermal processes may be written for a continuum as:

Mass:

$\nabla \cdot(\rho \tilde{V})=0$

Momentum:

$\rho \tilde{V} \cdot \nabla \tilde{V}=-\nabla p+\nabla \cdot(\mu \nabla \tilde{V})$

Energy:

$\rho C_{p} \widetilde{V} \cdot \nabla T=\nabla \cdot(k \nabla T)$

Here, $\mu$ is the dynamic viscosity and $\tilde{V}$ is the velocity vector. For a solid region, the conduction equation is used, given, with thermal conductivity of the solid $k$, as $\frac{\partial}{\partial x_{i}}\left(k \frac{\partial T}{\partial x_{i}}\right)=0$

For conjugate problems, the heat conduction in the solid region and the flow in the fluid region are generally solved separately and then coupled at the solid-fluid interface through the boundary conditions. Other approaches have also been developed. The energy input may be provided by chemical reactions, as is the case in combustion or chemical vapor deposition [5-6]. The coupled equations for the chemical species need to be solved in these cases.

Many numerical methods are available for solving the specific equations that may be derived from the preceding general equations for different processes and systems, such as energy systems, building fires, gas turbines and so on [7-8]. However, as mentioned earlier, it is very important to obtain accurate, realistic and reliable results that may form the basis for system design and optimization. However, several challenges are encountered in typical practical thermal systems and processes and must be addressed. Some of the main ones are:

\section{Variable material properties \\ Time-dependent phenomena \\ Accurate imposition of complicated boundary conditions \\ Complex domain \\ Combined and complex transport mechanisms \\ Verification and validation \\ Multiple length and time scales \\ Uncertainty in input data}

A few examples are considered to show the approaches to overcome these challenges and also present typical outputs from numerical simulation.

The validation of the mathematical and numerical model is a critical, though often difficult and involved task [9]. In most cases, considerable effort is directed at verification of the numerical scheme and validation of the model because of the critical importance of obtaining accurate results. Frequently, experimental systems are specially built to provide data for validation. In some cases, earlier numerical and experimental results may be available for comparisons with the current results for validation. Once the model has been validated and accurate results have been generated, the design, prediction and optimization of the system and process may be undertaken.

\section{DESIGN AND OPTIMIZATION}

The design of the thermal system can be carried out on the basis of the simulation results from a validated mathematical and numerical model. A detailed parametric study may be carried out, followed by a sensitivity analysis, to determine the important parameters. The appropriate parameters may be chosen to satisfy the given requirements, without violating the constraints. Typical constraints are posed on dimensions, flow rates, pressures and temperatures. An acceptable design is obtained if the requirements and constraints are satisfied. This is obviously not unique and a domain of acceptable designs is usually obtained. The basic process for obtaining and evaluating an acceptable design is discussed in detail in [1].

Following the development of acceptable, or feasible, designs, optimization of the thermal process and system is carried out to minimize or maximize a chosen objective 
function. Some of the objective functions that may be employed for thermal systems are:

1. Efficiency of the process

2. Energy and material consumption

3. Productivity or production rate

4. Environmental effect

5. Product quality

6. Capital and operational costs

7. Use of renewable energy sources

Obviously, several other similar objective functions can be considered. Many of these objectives overlap and one may focus on the dominant one or more objectives. In many cases, the demands posed by one objective function may counteract those posed by another. In manufacturing, for instance, product quality can often be improved by reducing the production rate. On the other hand, an increase in efficiency of a production facility or a power plant would enhance the output and also reduce the environmental effect. Therefore, the optimal design and operating conditions depend on the chosen objective function. Our focus here is reduction in energy and material consumption and in the environmental effect. However, the quality of the product, stability and consistency of the output, repeatability and reliability are also important considerations that need to be addressed for a realistic and useful optimization.

\section{TYPICAL RESULTS AND DISCUSSION}

\subsection{Isolated heat sources}

Some numerical and experimental results for different types of thermal systems are discussed in this section. Figure 2 (a) shows the calculated flow and temperature distributions in a channel containing two isolated thermal sources. A stationary, passive, vortex generator is placed upstream of the sources to generated vortices and thus enhance the heat transfer from the sources. The configuration is representative of an electronic system. The fluid is taken as air, the sources of copper and the channel walls of an insulating material like wood, so that material properties are reasonably well known. Since there is conduction heat transfer in the walls, a conjugate problem has to be solved. The time-dependent solution is needed to determine the disturbance frequency spectrum and match the dominant frequency due to the vortex generator with that due to the sources in order to obtain resonance and substantially enhance the heat transfer. High Reynolds number, Re, where Re is based on inlet velocity and channel height, leads to chaotic behavior and at still higher numbers to turbulence. The geometry is generally much more complex in practical systems like servers and data centers, requiring threedimensional solutions. Both experiments and numerical simulations may be employed to obtain the desired results for design and optimization [10].

With respect to optimization, the heat transfer rate should be maximized so that the energy consumed for generating the flow is minimized. This would also help with increasing the compactness of the packaging. As is well known, there are many ways to increase the heat transfer rates in the problem sketched in Figure 2 (a), including increased flow rate, decreased inlet temperature, and geometry variation. However, another critical aspect that must be included is the pressure needed for the flow. We cannot increase the heat transfer arbitrarily, without ensuring that the pressure drop in the channel is acceptable. A non-dimensional parameter $\Theta / \Delta P$, which represents the ratio of heat transfer to the pressure drop, is plotted against Re in Figure 2 (b). Both experimental data and numerical simulations are used. The maximum around 2 at $\mathrm{Re}=2300$ represents the optimum, which satisfies the constraints on the flow rate, given in terms of Re being greater than about 1000 . At very low flow rates, $\Delta \mathrm{P}$ is very small or close to zero, making the optimization parameter very high even though the heat transfer is still small.
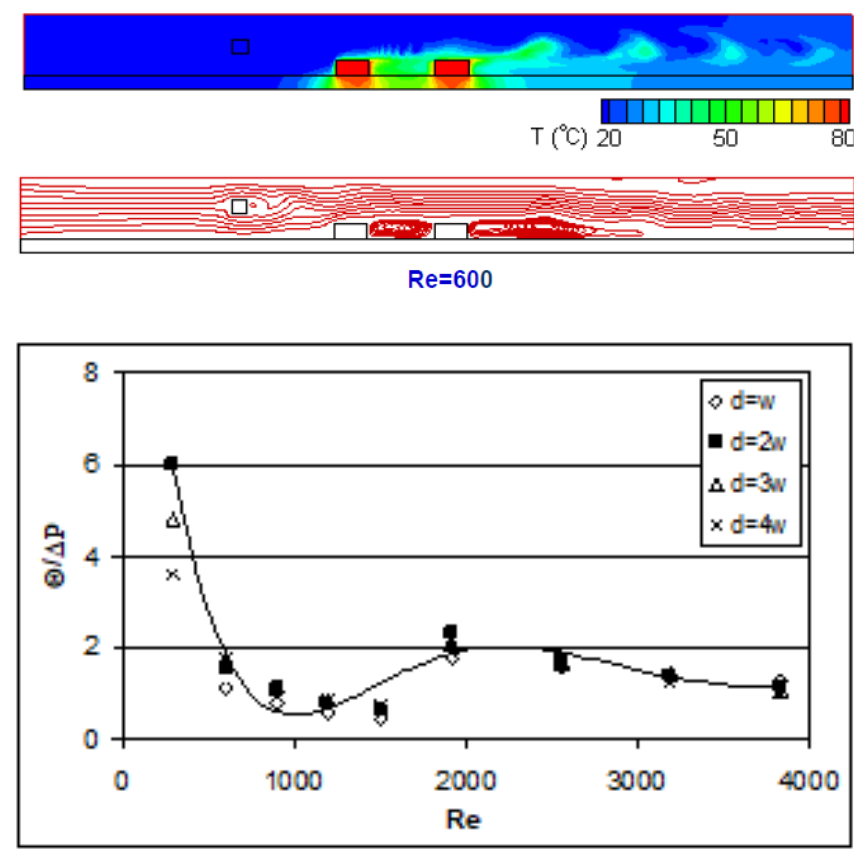

Figure 2. (a) Flow and temperature distributions in a channel with isolated sources and a vortex generator; (b) Optimal conditions for heat removal without the vortex generator

However, this optimization parameter is an arbitrary combination of two objectives: heat transfer rate and pressure drop. It is better to consider the two objectives separately and draw the envelope of non-dominant solutions. This envelope is known as the Pareto front, as mentioned earlier. Figure 3 shows the results of such a multi-objective optimization, considering the two aspects, heat transfer rate and pressure separately to generate a Pareto frontier [11]. The corresponding response surfaces are also shown in terms of two length dimensions that define the locations of the two sources. The heat transfer is given in terms of the Stanton number, $\mathrm{St}$, which is Nusselt number, $\mathrm{Nu}$, divided by Reynolds number, Re, and Prandtl number, Pr. The Pareto frontier may be used to trade-off between the two objectives to obtain the final design.

Now considering the case with a vortex generator, a similar approach may be used. The results obtained from a composite objective function, $\mathrm{F}$, which includes heat transfer rates from the two sources, $\mathrm{Q}_{1}$ and $\mathrm{Q}_{2}$, as well as the pressure needed, are shown in Table 1 . This objective function is again arbitrary and is based on the knowledge about the given system. It is seen from the table that the dimensions and shape of the optimum vortex generator can be obtained for a variety of conditions. 


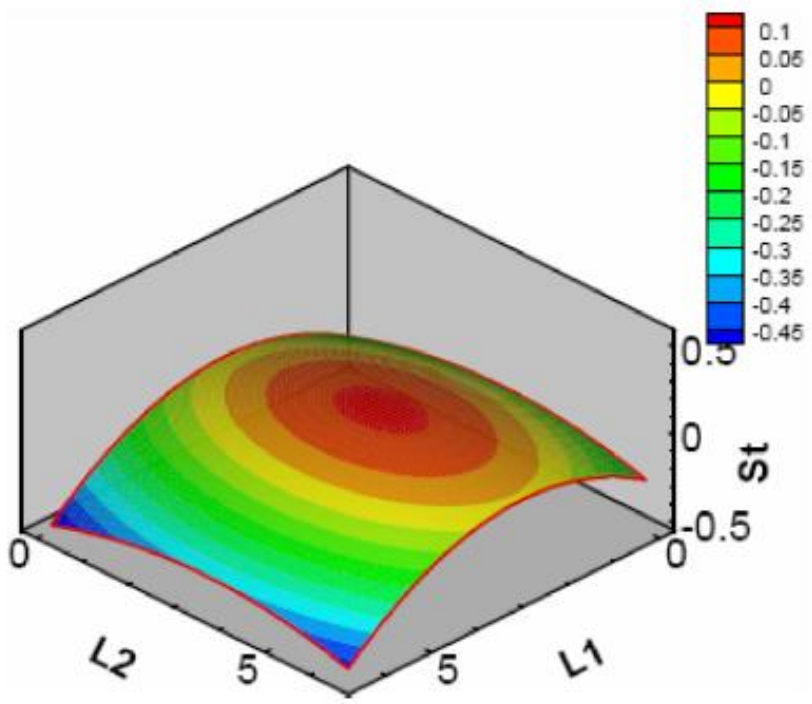

Table 1. Optimization of a vortex promoter in a channel with two isolated heat sources

F $=W_{1} \bar{Q}_{1}+W_{2} \bar{Q}_{2}-W_{3} \overline{\Delta P}$
\begin{tabular}{|c|c|c|c|}
\hline & Geometry & $R e^{*}$ & $h_{p}{ }^{*} / H$ \\
\hline$W_{1}=1, W_{2}=W_{3}=0$ & Hexagonal & 5600 & 0.47 \\
\hline$W_{2}=1, W_{1}=W_{3}=0$ & Circular & 5600 & 0.36 \\
\hline$W_{3}=1, W_{1}=W_{2}=0$ & No vortex promoter & & \\
\hline$W_{1}=W_{2}, W_{3}=0$ & Hexagonal & 5600 & 0.47 \\
\hline$W_{1}=W_{2}=W_{3}$ & Square & 5600 & 0.16 \\
\hline$W_{1}=W_{2}=2 \times W_{3}$ & Circular & 5600 & 0.47 \\
\hline$W_{1}=W_{2}=3 \times W_{3}$ & Hexagonal & 5600 & 0.47 \\
\hline$W_{1}=W_{2}=W_{3} / 2$ & No vortex promoter & & \\
\hline$W_{1}=W_{2}=W_{3} / 3$ & No vortex promoter & & \\
\hline
\end{tabular}

\subsection{Data centers}

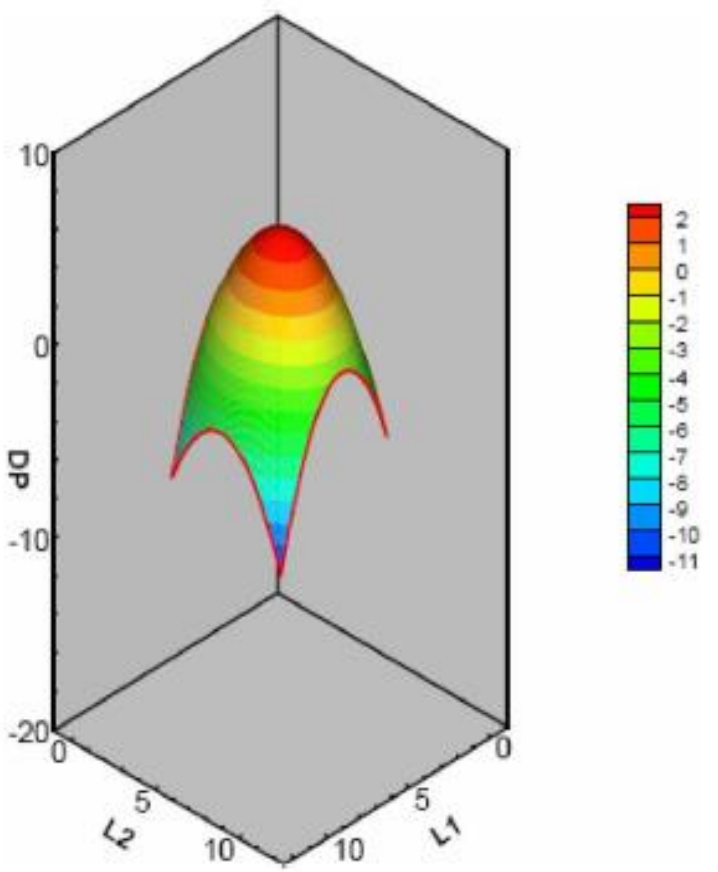

The thermal management of data centers is an important consideration since the cooling often requires considerable amount of energy, very much like power plants [12]. Modeling and numerical simulation can be used to obtain the flow and temperature fields. Figure 4 shows a typical data center, along with the flow and temperature distributions for two locations of the outlet. These results can be used to pinpoint hot spots and areas of low heat transfer, so that the system may be designed to meet the stringent temperature constraints of electronic systems that typically require temperatures to be lower than about $80{ }^{\circ} \mathrm{C}$. The optimization involves choosing the geometry and operating conditions, particularly the flow rate and inlet temperature, in order to minimize the energy consumed. Chillers are needed to lower the temperature below the ambient and considerable energy is saved if chillers are not employed and ambient air is used for heat removal. But this requires a consideration of heat input due to the electronic system, possible hot spots and effectiveness of the circulating flow.

Another interesting consideration arises with respect to the effect of the environment on the thermal energy needed for cooling of a data center. Frequently, several data centers are available for a given organization, spread out over the country or the world. One could then vary the load, depending on the environmental conditions to minimize the power consumption [13-14]. Figure 5 shows a typical data center and the calculated results for different loads. Thus, colder regions may be effectively employed in the summer and warmer ones in the winter without the extensive use of the power consuming chillers to cool the air entering the data center. The electronic load could be kept low in order to cool the system with the use of a simple fan. At larger loads, the chiller will be needed. The load and location of the data center can be optimized in order to minimize the power consumption. This will also substantially reduce the effect on the environment. Figure 6 shows these effects quantitatively by considering two locations: Princeton and Seattle in USA. A higher load and higher ambient temperature demand higher power consumption, as expected.

Figure 3. Response surfaces and Pareto frontier for the optimization of an electronic system represented by two isolated heat sources in a channel without a vortex generator 


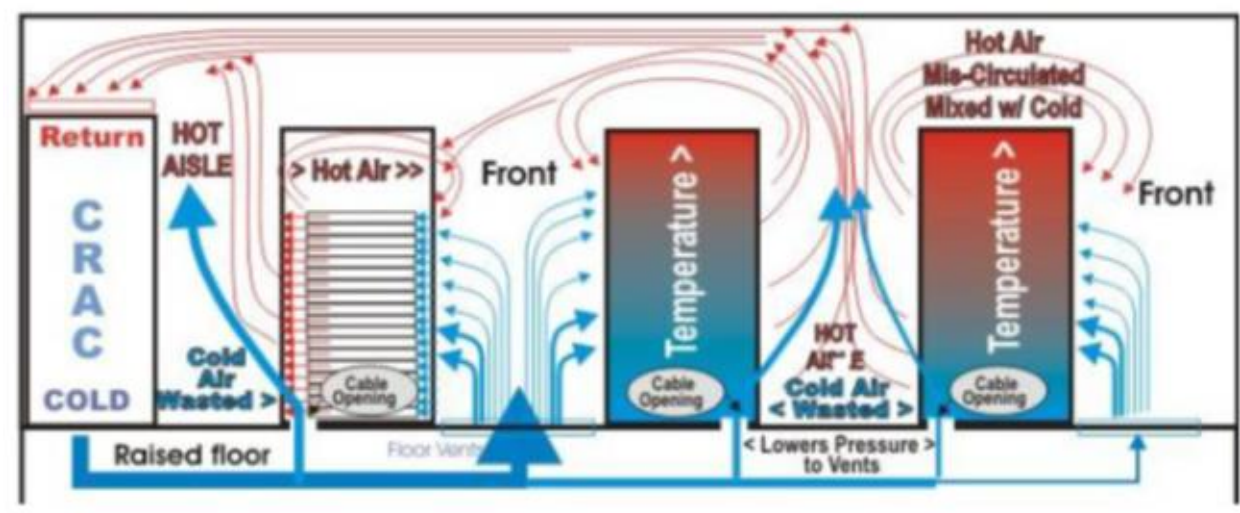

(a)

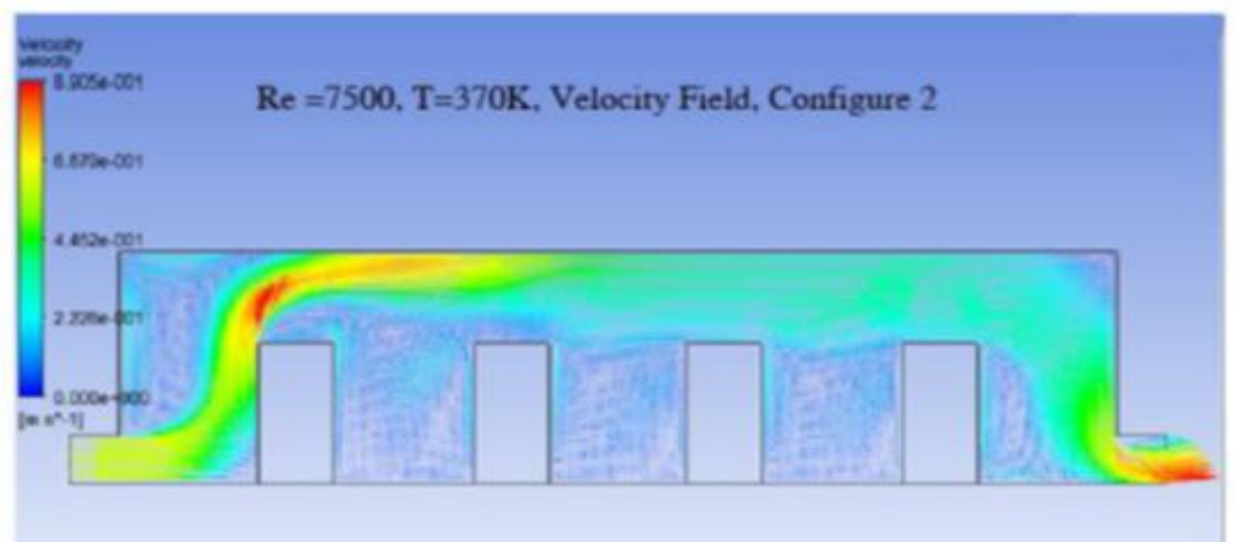

(b)

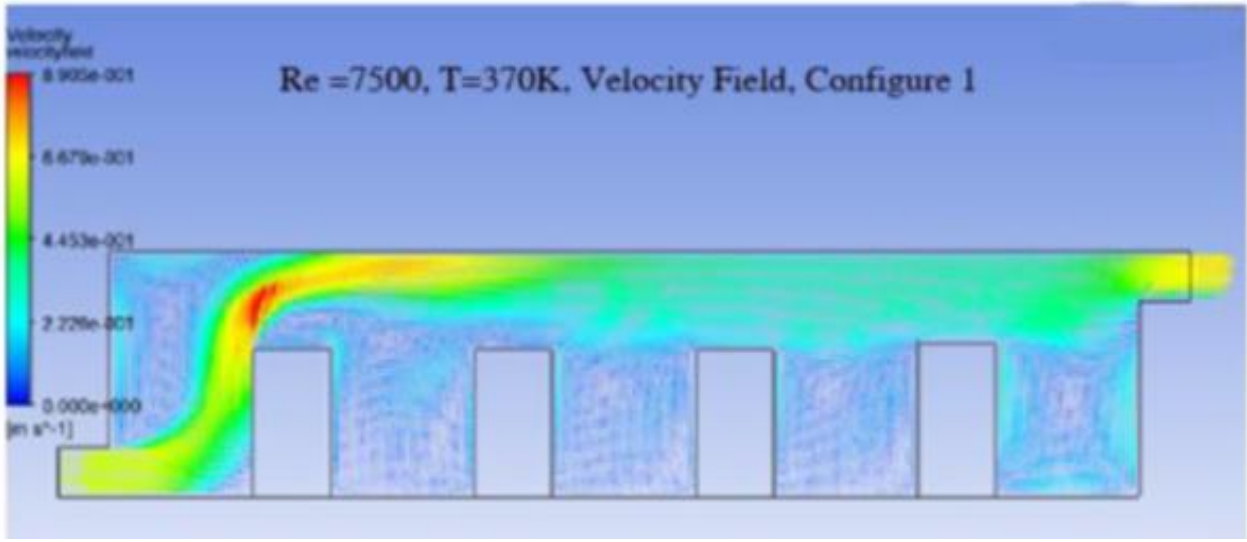

(c)

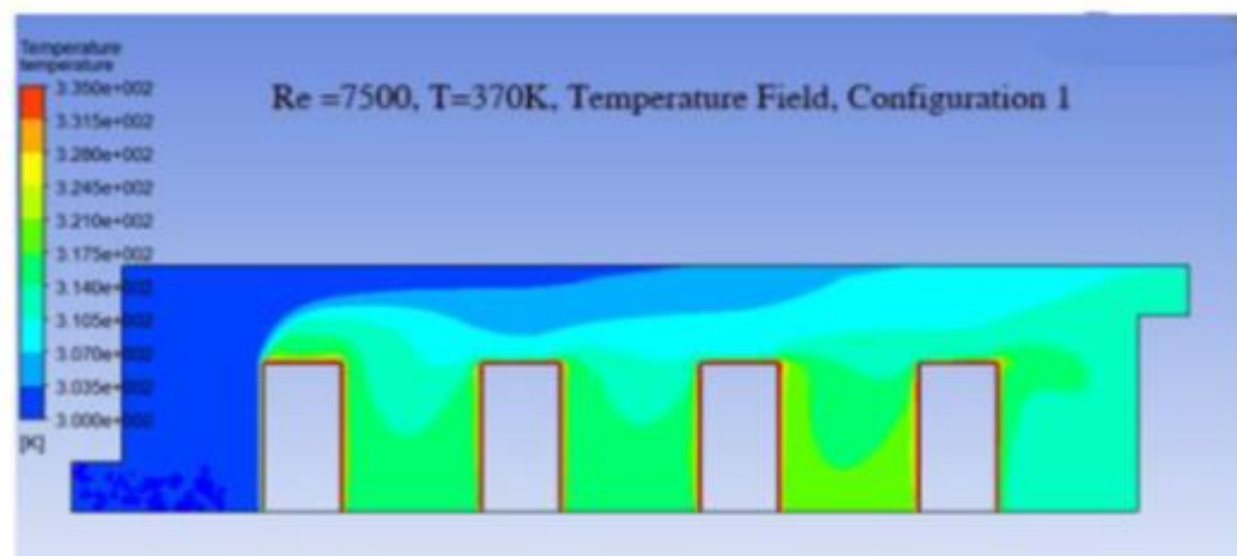

(d)

Figure 4. A typical data center and calculated velocity and temperature distributions for two configurations of the outlet 

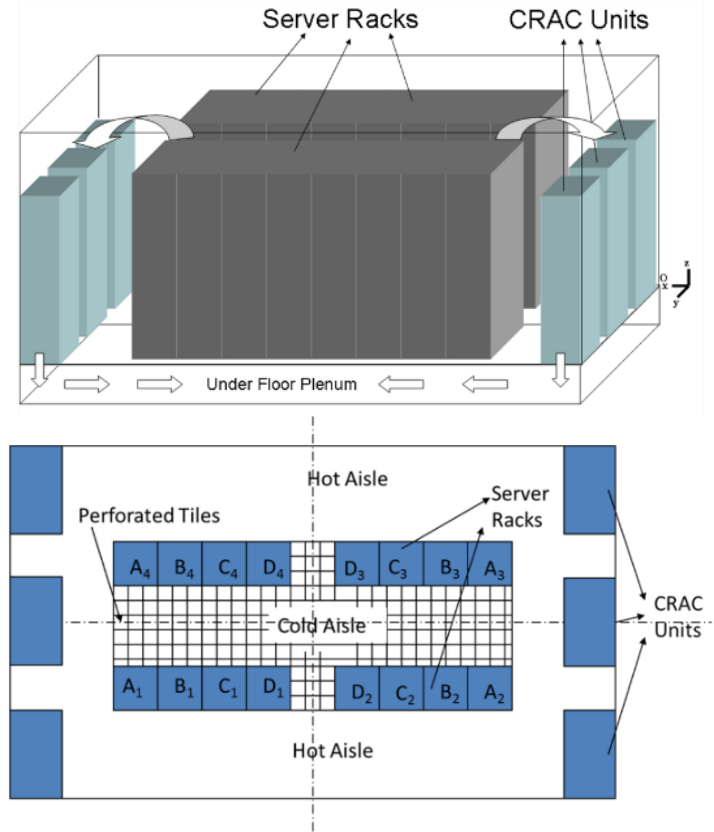

(a)

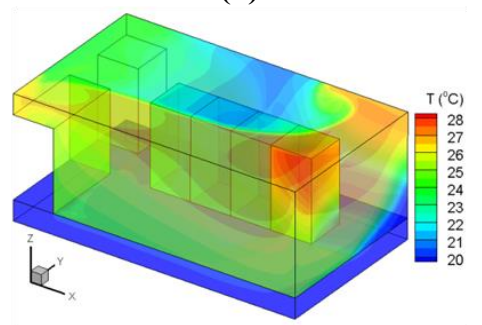

(b)
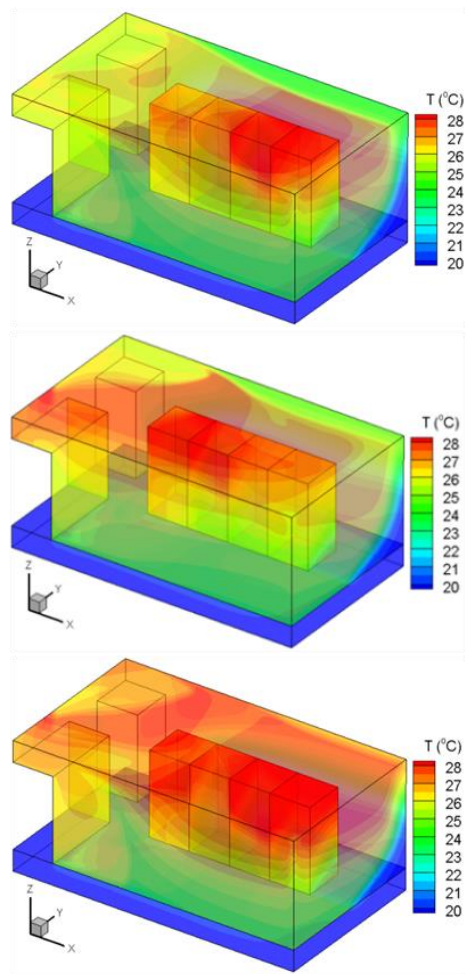

(c)

Figure 5. (a) Sketch of the data center considered; (b) and (c) Calculated flow and temperature fields in the data center. The load is $25 \%$ of the maximum in one server in the first figure and then increases to $50 \%$ with different servers being active. The last one has the largest heat input in the system
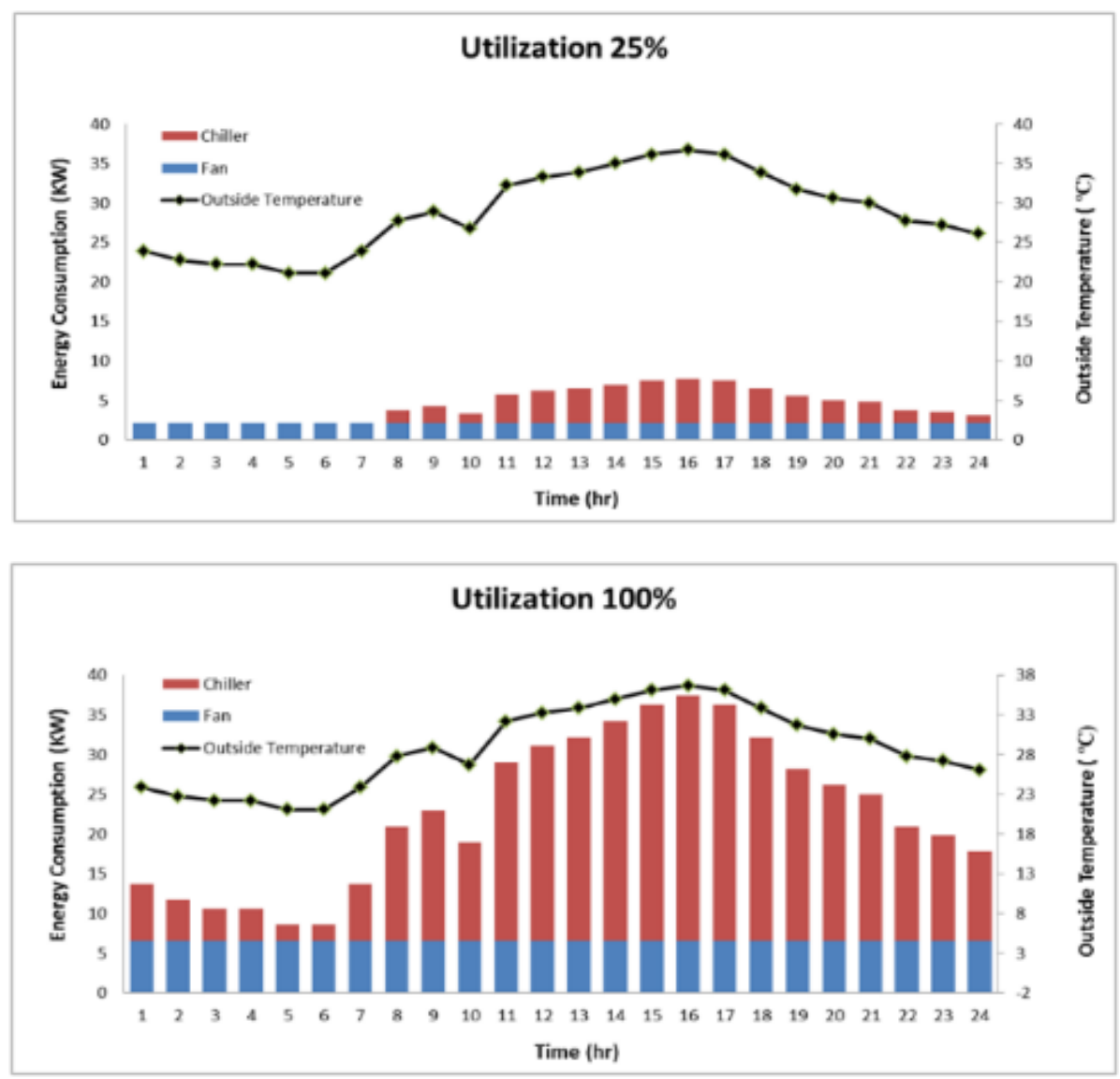

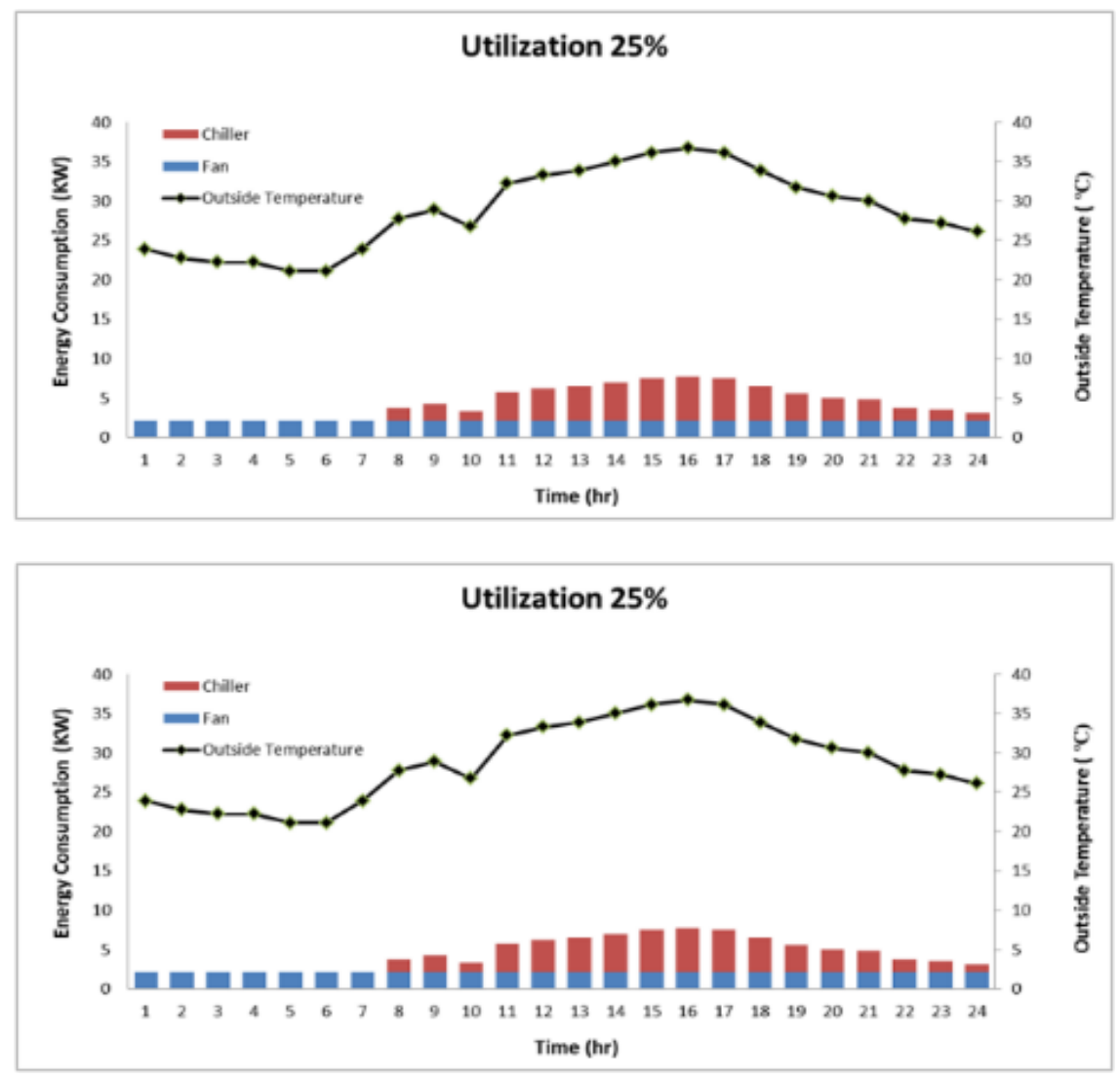

Figure 6. Effect of environmental conditions and load on power consumption for cooling of data centers.

Top: Princeton, NJ, in August and Bottom: Seattle, WA, in January. The red portion represents the power consumed by chillers

\subsection{Optical fiber drawing}

Let us now consider the optical fiber drawing process, sketched in Figure 7 (a). The draw furnace as well as cooling and coating processes are shown. Figure 7 (b) shows the hightemperature furnace that heats up the glass preform to its softening point of about $1900{ }^{\circ} \mathrm{C}$, allowing it to be drawn into an optical fiber of diameter around $125 \mu \mathrm{m}$. The material properties of glass vary strongly with temperature $\mathrm{T}$, particularly its viscosity $v$ which varies exponentially with temperature. A commonly used expression in S.I. units is given in terms of the temperature $\mathrm{T}$ and melt or softening temperature $\mathrm{T}_{\text {melt }}$ is:

$$
v=4545.45 \exp \left[32\left(\frac{\mathrm{T}_{\text {melt }}}{\mathrm{T}}-1\right)\right]
$$

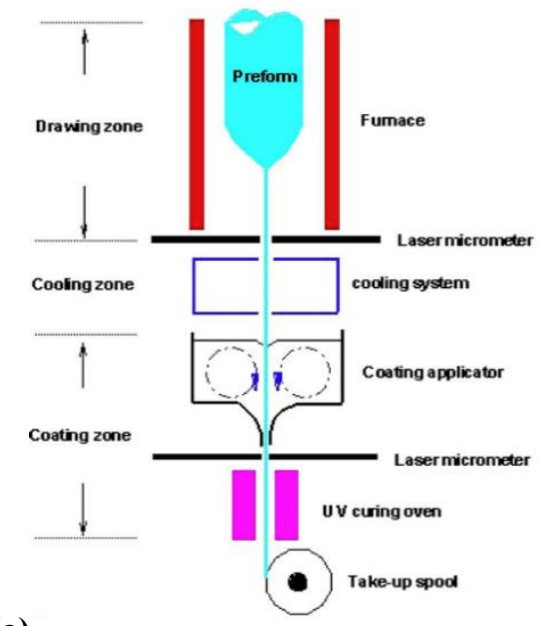

(a)$$
\text { a) }
$$

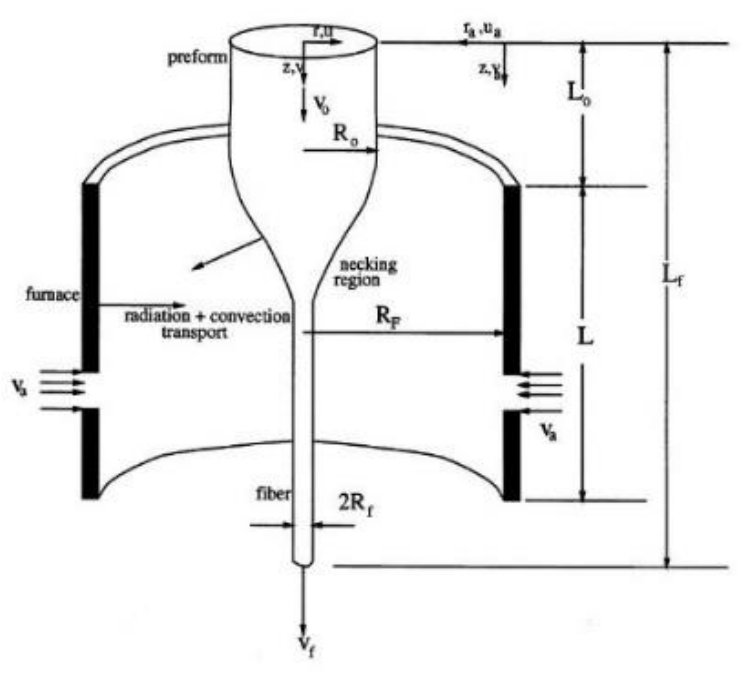

(b)

Figure 7. Optical fiber drawing process; (a) Hightemperature furnace for heating and drawing the silica glass preform into an optical fiber

All heat transfer mechanisms arise, though radiation is the dominant mode. The modeling is quite complicated and involves fine grids to capture the temperature variations across the preform/fiber [15]. The flow in the glass is computed and the free surface is obtained from a balance of the forces acting on it. This calculation yields the profile as the glass goes from a diameter of around $10 \mathrm{~cm}$ of the preform to $125 \mu \mathrm{m}$ in a relatively short distance of about $30 \mathrm{~cm}$ in the furnace. If the fiber is drawn at low furnace temperature or at high speed, it would break due to viscous rupture. A feasible domain is 
determined from the numerical calculations and confirmed by experiment [16].

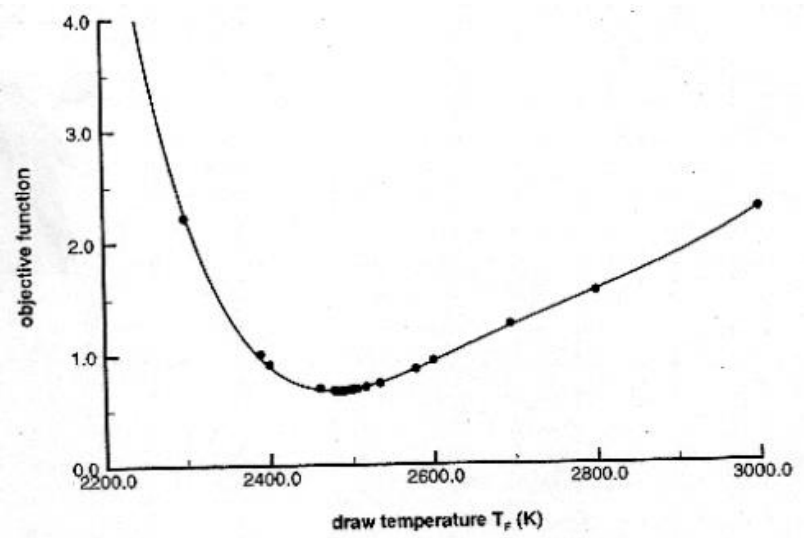

(a)

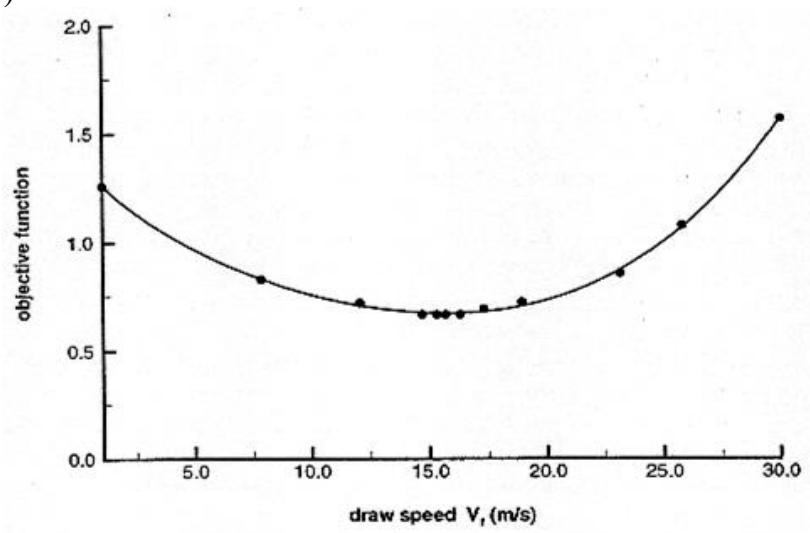

(b)

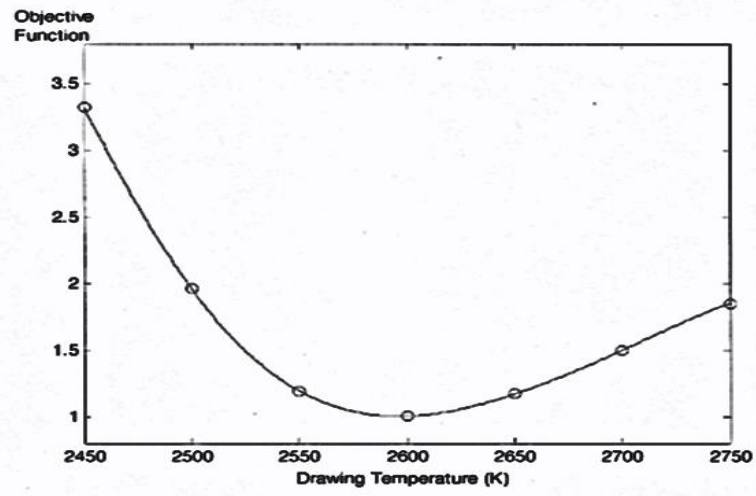

(c)

Figure 8. Optimization of the optical fiber drawing process in terms of main operating parameters. (a) Draw temperature;

(b) Fiber speed; (c) Results for hollow fiber. The objective function represents fiber quality per unit cost

The simulation results obtained from the validated numerical model may be used to optimize the process. The objective function could be based on thermally induced defects, tension in the fiber, velocity difference across the fiber, and draw speed. The first three relate to fiber quality and the last one to the production rate. A higher production rate is desirable to reduce the energy consumption and lower costs. Similarly, higher fiber quality reduces wastage and again saves costs and energy. All these quantities may be combined into a single composite objective function [17]. Otherwise, mutiobjective optimization, leading to Pareto frontiers, may be undertaken. The smallest value of the composite objective function indicates the smallest amount of defects, or higher fiber quality, per unit cost. The furnace temperature and fiber speed are taken as the two main variables to increase the efficiency and lower costs. These variables are considered separately and the results indicate the optimal conditions to obtain the best quality fiber per unit cost. Figure 8 shows the resulting optima, using a multivariate search scheme. Similarly, production rate can be considered as another objective function and the process optimized to obtain the highest rate of production, while keeping the quality as acceptable. Figure 8 (c) shows the results for hollow fiber drawing.

\subsection{Chemical vapor deposition}

Another important material processing problem is shown in Figure 9. This is the widely used chemical vapor deposition (CVD) system for fabricating thin films used in electronics, photovoltaics and optical applications [18-19]. The films are deposited on a chosen heated substrate, such as sapphire and silicon carbide, from chemical reactions in the gases and at the surface. The simulation is complicated due to the typically large number of intermediate species and reactions that must be included. Two configurations are shown in Figure 9. In many cases, the susceptor is rotating and gases impinge on it, resulting in a $3 \mathrm{D}$ problem. Conjugate transport is obviously needed. The film thickness starts at the nanometer level and the final thickness is typically 10-20 microns. Since the manufacturing system is at the macro or engineering scale, a multi-scale problem has to be solved. Validation is needed and well controlled experiments are performed to obtain the data for comparison. It is important to have excellent film uniformity and thickness.

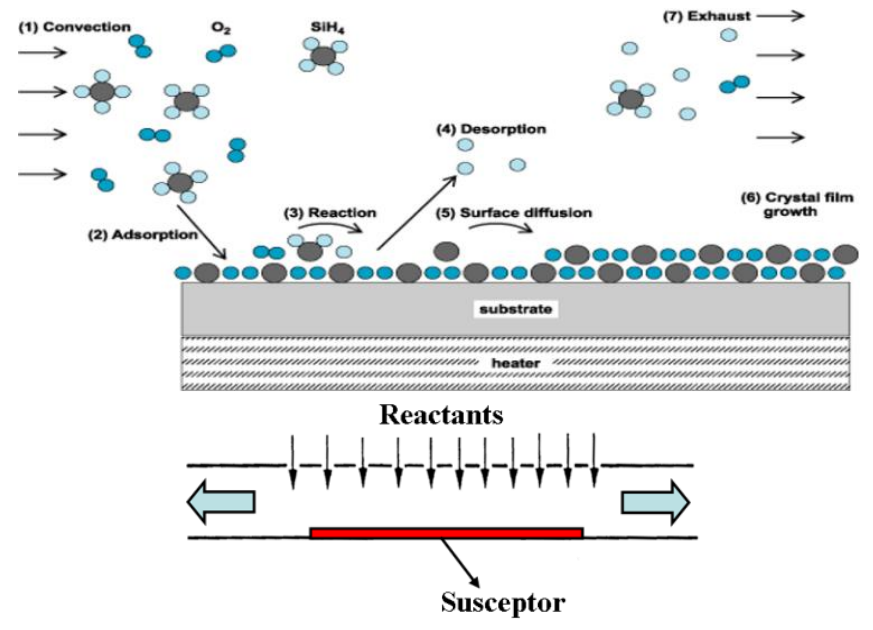

Figure 9. (a) A horizontal CVD reactor for thin film fabrication; (b) A vertical impinging reactor

Figure 10 shows some typical results for an impinging reactor such as the one shown in Figure 9 (b). Optimization of this system is very important since the gases are expensive and energy consumption is high. A reduction in energy needed to heat the susceptor and in the amount of gases lost in the process are important considerations. The end portions of the film, shown in red in the simulation, have to be discarded if the thickness is not up to the desired level. If the thickness is not uniform or deposition is not satisfactory, the percentage area of the film that may be used in device manufacture is 
reduced. This percentage working area (PWA) can be taken as the objective function and the inlet velocity $\mathrm{V}$ and susceptor temperature $\mathrm{T}$ as the two main variables.

Figure 11 shows the response surface generated for this problem for silicon deposition on the basis of simulation results and the optimum point, which is also shown on a twodimensional plot. Clearly, a high PWA increases the efficiency of the processes and reduces the energy consumed. It also reduces the effect on the environment due to the gases lost from the system. Other objective functions have also been considered.

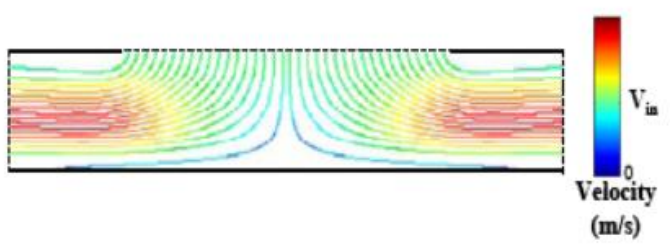

(a)

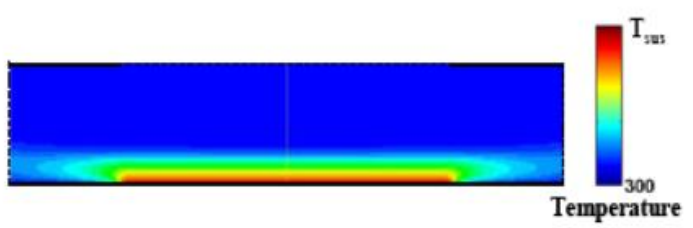

(K)

(b)

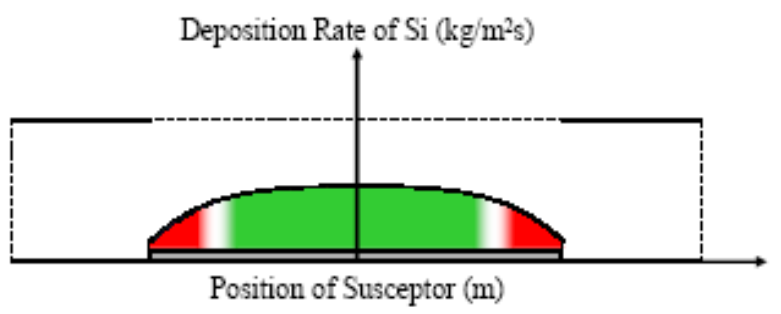

(c)

Figure 10. Typical results from an impingement CVD reactor with stationary deposition surface, or susceptor. (a) Flow; (b) Temperature distributions; (c) The deposited film

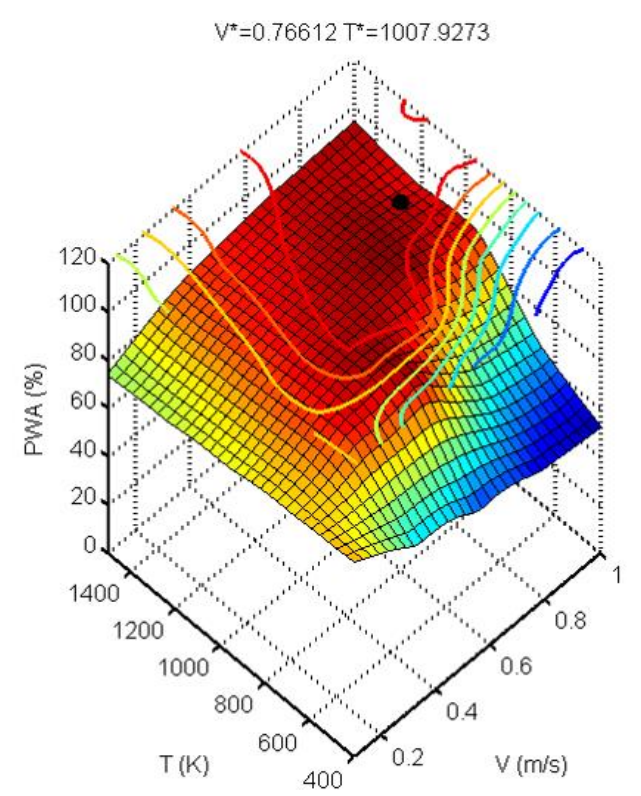

(a)

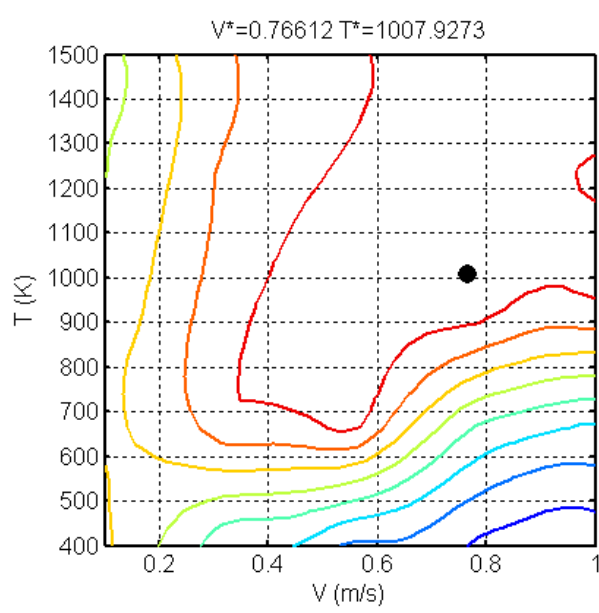

(b)

Figure 11. (a) Response surface and optimal point to achieve maximum percentage working area in a typical CVD reactor for depositing Silicon; (b) Optimal point on contour plot.
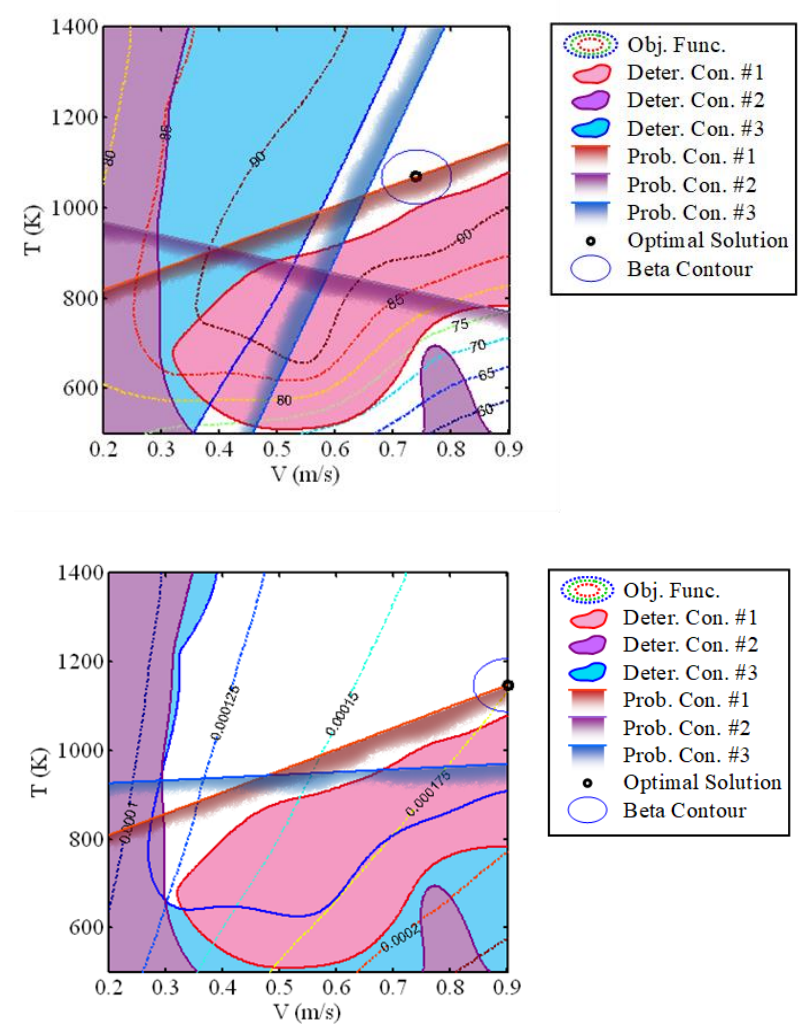

Figure 12. Optimization with uncertainties in the operating conditions for the CVD reactor considered in Figure 11

In such complex processes, uncertainties arise in the design parameters and operating conditions. These are important and must be included to obtain the final, realistic, optimal design. Figure 12 shows the calculated results on optimization of the CVD reactor considered for Figure 11 in the presence of uncertainties in the inlet velocity $\mathrm{V}$ and susceptor temperature T. Several deterministic and probabilistic constraints are considered here. The variables are also not taken as fixed, but as probabilistic with a normal distribution. The spread of these variables is seen in Figure 12. The optimal point moves away from that obtained from a deterministic model with fixed operating conditions. This is an important consideration in 
obtaining realistic and practical results [20].

\subsection{Heat Rejection Systems}

A particularly important thermal system with respect to environmental effects is concerned with heat rejection from a power plant to a water body, such as a cooling pond, lake, river or ocean. The water from the condensers of a power plant are often cooled by discharge into and intake from a natural lake or pond [21-22]. The flow causes enhanced mixing, as well heat input into the lake. The overall effect is to alter the annual thermal cycle of the lake and increase the temperatures in order to lose the additional energy. Most of the energy is lost at the surface and only a small amount at the sides. Figure 13 (a) shows a sketch of the system and the numerical results on a deep natural lake in New York state, along with experimental data. The lake is stratified over the summer and has a uniform temperature distribution in the winter. The results obtained with a thermal discharge from a large power plant are shown in Figure 13 (b). The temperatures are seen to rise by a few degrees and the thermal cycle is shifted by several days, particularly the onset of summer stratification. These effects, though seemingly modest, accumulate over the years and serve to destroy organic life in the lake and thus are unacceptable.
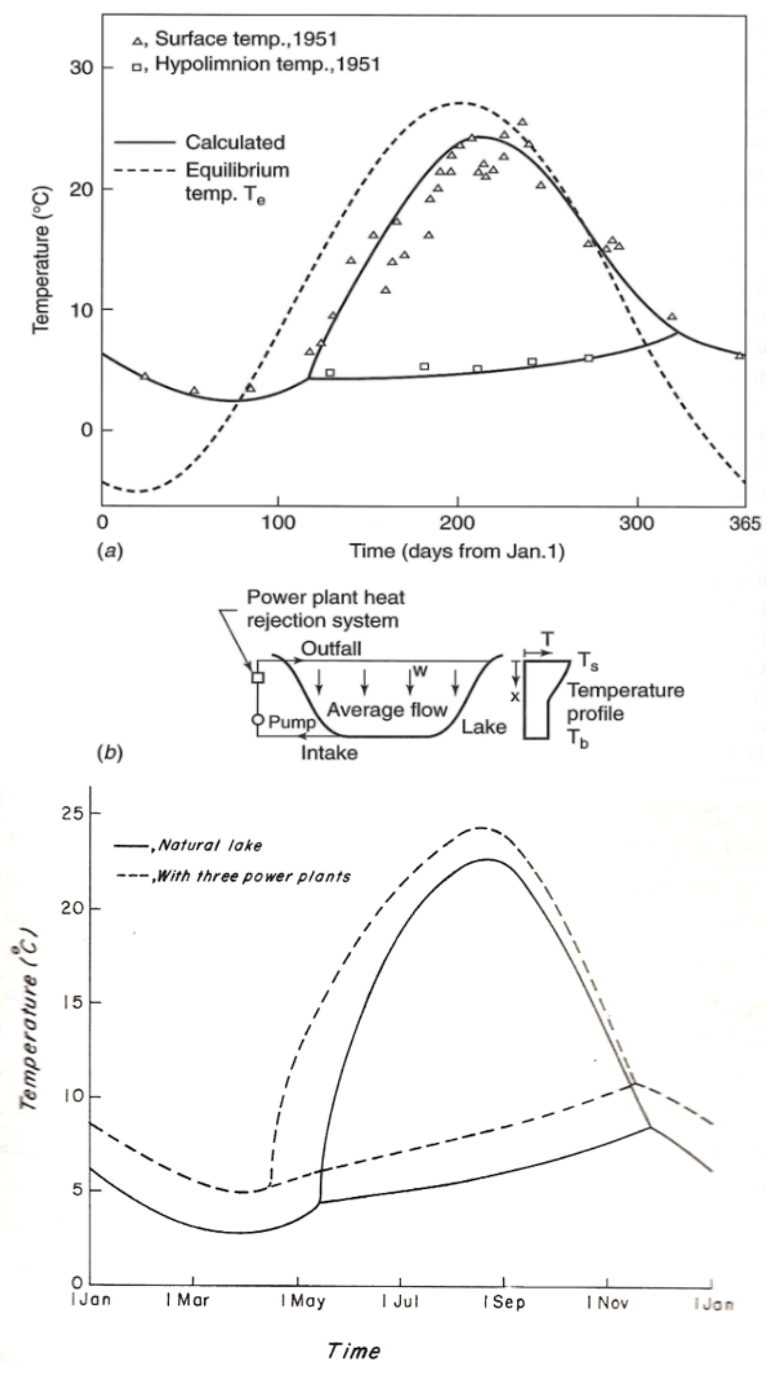

Figure 13. (a) System for heat rejection from a power plant to a lake and the calculated temperature cycle of a natural lake, along with experimental data; (b) Effect of thermal discharge into the lake on the thermal cycle

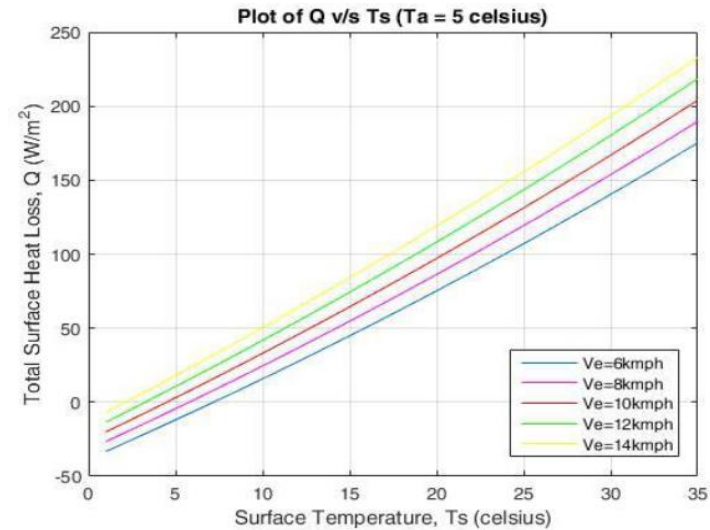

(a)

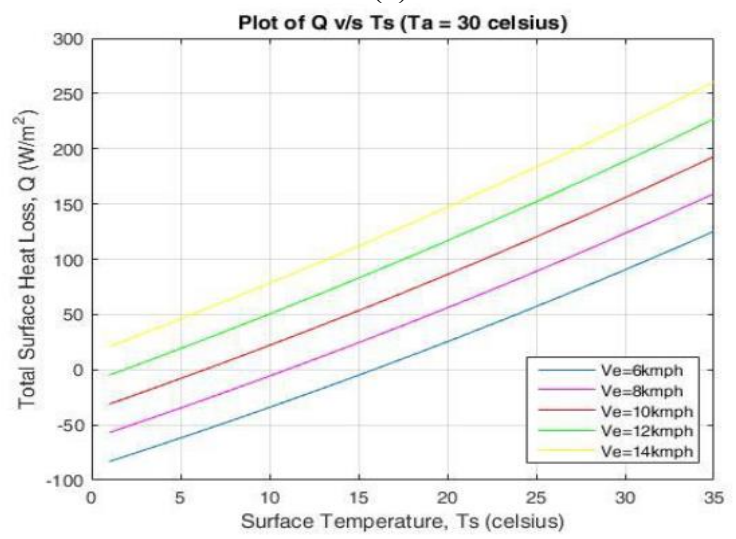

(b)

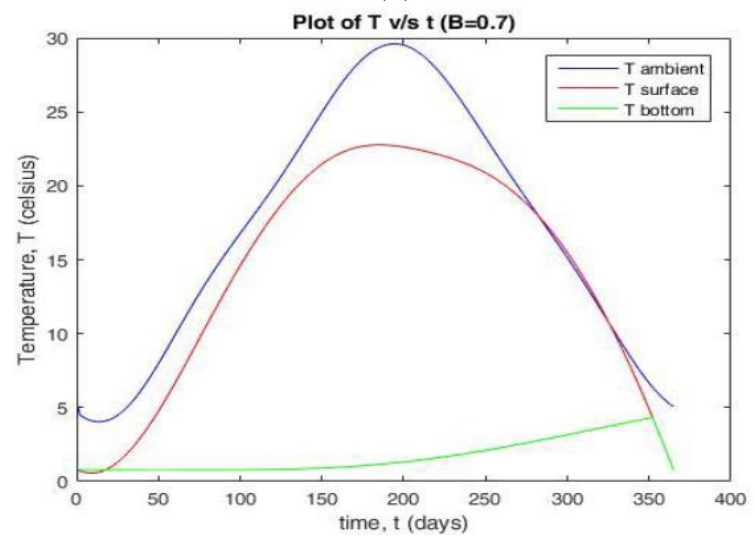

(c)

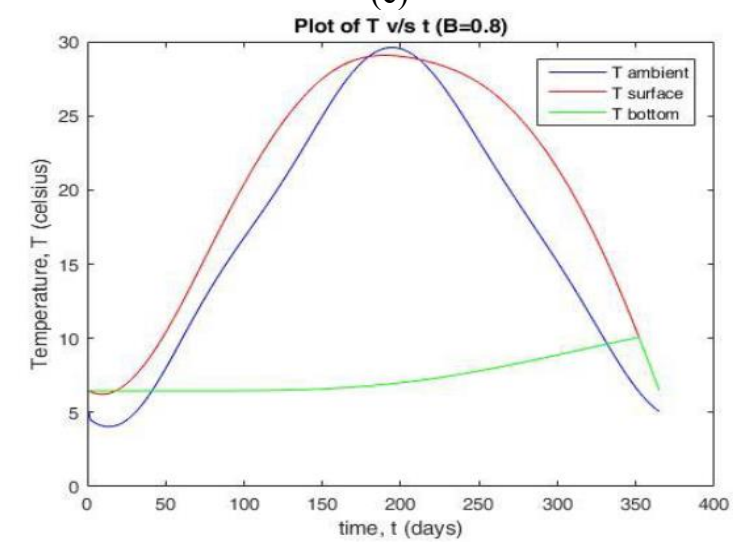

(d)

Figure 14. (a) and (b) Surface heat loss from a lake in New Brunswick, NJ, USA, as a function of the surface

temperature in winter and summer, respectively; (c) and (d) Resulting temperature cycle if the back radiation is reduced, as given by the cloud cover parameter $\mathrm{B}$, a larger value indicating smaller back radiation 
The heat gained by the lake is largely due to solar flux at the surface and the energy lost mainly due to evaporation, convection heat loss and back radiation. All these mechanisms can be modeled for a given location if the ambient conditions are known. The total energy lost is shown in Figures 14 (a) and (b) as a function of the surface temperature at two ambient temperatures, $5{ }^{\circ} \mathrm{C}$ and $17.5{ }^{\circ} \mathrm{C}$, representing winter and summer, respectively, in New Brunswick, NJ [23]. Steady state conditions are given by zero heat loss. But due to the transient behavior of the water body, these conditions are never attained. Instead, a heat transfer coefficient with respect to the equilibrium or steady-state temperature may be defined and calculated for use in the energy transfer calculations, as done in Ref. [21].

The effect on the temperature cycle is shown in Figures 14 (c) and (d) for two circumstances of back radiation. A higher value of the parameter B represents larger cloud cover or less back radiation. This would also apply for larger concentration of greenhouse gases, so that the back radiation is reduced. The effect is higher temperatures if back radiation is curbed, as expected. These results can be used to determine the environmental effects of heat rejection and modify the discharge temperature and flow rate to minimize the effects.

\section{CONCLUDING REMARKS}

Thermal processes and systems are of interest in a wide range of applications, from manufacturing and electronics to heat rejection. It is important to increase the efficiency and product quality, thus resulting in reduced energy consumption and smaller environmental effect. The mathematical modelling and numerical simulation are complicated since they need to address several challenges, such as material property variation, validation, complex domains, combined mechanisms, intricate operating conditions, uncertainties, and multiple scales, in order to obtain accurate, realistic and reliable results. These results form the basis for prediction and control of the process. They are also essential for design and optimization. It is also crucial to optimize these systems and processes to improve the output in terms of product quality, efficiency and effectiveness, while reducing the effect on the environment. This paper presents approaches that may be adopted and discusses several important examples to demonstrate the methodology and results from such optimization.

\section{ACKNOWLEDGMENTS}

The author acknowledges the support provided by the National Science Foundation and industry for the work presented here. The work of several students and collaborators is also acknowledged.

\section{REFERENCES}

[1] Jaluria Y. (2008). Design and optimization of thermal systems. Second Edition. CRC Press, Boca Raton, Florida.

[2] Bejan A, Tsatsaronis G, Moran M. (1996). Thermal Design and Optimization. John Wiley \& Sons, New York.

[3] Deb K. (2002). Multi-objective optimization using evolutionary algorithms. John Wiley \& Sons, New York NY.

[4] Jaluria Y. (2003). Thermal processing of materials: From basic research to engineering. J. Heat Transfer 125: 957979. http://dx.doi.org/10.1115/1.1621889

[5] Mahajan RL. (1996). Transport phenomena in chemical vapor deposition systems. Advances in Heat Transfer 28: $339-425$. http://dx.doi.org/10.1016/S00652717(08)70143-6

[6] Jaluria Y. (2018) Advanced materials processing and manufacturing. Springer, Cham, Switzerland. https://doi.org/10.1007/978-3-319-76983-7

[7] Jaluria Y, Torrance KE. (2003). Computational heat transfer. Second Edition. Taylor \& Francis Pub. Co., New York.

[8] Minkowycz WJ, Sparrow EM, Murthy J. (Eds). (2006). Handbook of numerical heat transfer. John Wiley \& Sons, New York.

[9] Roache PJ. (1998). Verification and validation in computational science and engineering. Hermosa Publishers, Albuquerque, New Mexico.

[10] Icoz T, Jaluria Y. (2004). Design of cooling systems for electronic equipment using both experimental and numerical inputs. J. Elect. Pkg. 126: 465-471. http://dx.doi.org/10.1115/1.1827262

[11] Zhao H, Icoz T, Jaluria Y, Knight D. (2007). Application of data driven design optimization methodology to a multi-objective design optimization problem. J. Eng. Design 18: 343-359. http://dx.doi.org/10.1080/09544820601010981

[12] Joshi Y, Kumar P. (Eds). (2012). Energy efficient thermal management of data centers. Springer, New York. http://dx.doi.org/10.1007/978-1-4419-7124-1

[13] Le K, Zhang J, Meng J, Bianchini R, Jaluria Y, Nguyen TD. (2011). Reducing electricity cost through virtual machine placement in high performance computing clouds, SC11 (Int. Conf. High Perf. Comput., Networking, Storage \& Anal.) Conference, Seattle, WA, USA.

[14] Zhang J, Jaluria Y. (2017). Steady and transient behavior of data centers with variations in thermal load and environmental conditions. Int. J. Heat Mass Transfer, 108:

374-385.

http://dx.doi.org/10.1016/j.ijheatmasstransfer.2016.12.0 28

[15] Paek UC. (1999). Free drawing and polymer coating of silica glass optical fibers. Journal of Heat transfer 121: 774-788. http://dx.doi.org/10.1115/1.2826066

[16] Roy Choudhury S, Jaluria Y, Lee SHK. (1999). A computational method for generating the free-surface neck-down profile for glass flow in optical fiber drawing. Numerical Heat Transfer 35: 1-24.

[17] Cheng X, Jaluria Y. (2005). Optimization of a thermal manufacturing process: Drawing of optical fiber. International Journal of Heat and Mass Transfer 48: 3560-3573

http://dx.doi.org/10.1016/j.ijheatmasstransfer.2005.03.0 12

[18] Jensen KF, Einset EO, Fotiadis DI. (1991). Flow phenomena in chemical vapor deposition of thin films. Ann. Rev. Fluid Mechanics 23: 197-232. http://dx.doi.org/10.1146/annurev.fl.23.010191.001213

[19] Eversteyn FC, Severin PJW, Brekel CHJ, Peek HL. (1970). A stagnant layer model for the epitaxial growth 
of silicon from silane in a horizontal reactor. J. Electrochem. Soc. 117: 925-931. http://dx.doi.org/10.1149/1.2407685

[20] Lin PT, Gea HC, Jaluria Y. (2010). Systematic strategy for modeling and optimization of thermal systems with design uncertainties. Frontiers in Heat Mass Transfer 1: 013003-1-20. http://dx.doi.org/10.5098/hmt.v1.1.3003

[21] Moore FK, Jaluria (1972). Thermal effects of power plants on lakes. Journal of Heat Transfer 94: 163-168. http://dx.doi.org/10.1115/1.3449888
[22] Kirillin G, Shatwell T, Kasprzak P. (2013). Consequences of thermal pollution from a nuclear plant on lake temperature and mixing regime. Journal of Hydrology 496:

$47-56$. http://dx.doi.org/10.1016/j.jhydrol.2013.05.023

[23] Bharadwaj N. (2016). A numerical study of the local warming of New Brunswick, NJ, due to thermal effects on local water bodies. M.S. Thesis, Rutgers Univ., New Brunswick, NJ. 